\title{
ACCURACY AND NUMERICAL EFFICIENCY OF APPROXIMATE METHOD FOR COMPLEX EIGENSOLUTION OF STRUCTURE WITH SPECIALIZED DAMPING DEVICE
}

\begin{abstract}
S. Hračov ${ }^{*}$
Abstract: The paper provides a detailed parametric analysis of the recently proposed approximate method for complex eigensolution of non-classically damped structures. This method is based on a combination of perturbation and incremental approaches and is applicable to the classically damped structures equipped with a specialized passive damping device. The numerical efficiency in terms of the required calculation times and the accuracy is demonstrated on a model of an existing structure for various combination of computational parameters. Wide numerical simulations revealed the selection of real eigenmodes of undamped system used in the approximate eigensolution as the most important parameter affecting the final accuracy.
\end{abstract}

Keywords: non-classical damping, complex eigensolution, perturbation method, passive damper

\section{Introduction}

The installation of the specialized damping device into the structure in order to suppress excessive vibration caused by e.g. wind or earthquake usually significantly changes the dissipative character. An assumption of the entire system being clasically damped is no longer valid and thus the normal mode method does not provide neither simplified solution of modal properties nor the response from the individual uncoupled differential equations. The decoupling of the equations of such a non-classically damped system is carried out with the use of a complex eigensolution. The complex eigenvalue problem is usually solved by the exact state-space method that is related to an increase in computational costs, since it requires the solution of the system of doubled size. These high numerical demands have motivated many authors during last decades to develop more efficient procedures see, e.g., (Claret, 1991; Adhikari, 2011).

In this paper, the numerical efficiency of the approximate method for complex eigensolution recently proposed by Hračov (2018) is presented by means of parametric analysis. The method is applicable to classically damped structures equipped with one passive damping element. The damping matrix of such system is assumed in the form

$$
\mathbf{C}=\mathbf{C}_{p}+\mathbf{d d}^{T} .
$$

Matrix $\mathbf{C}_{p}$ represents the classical part of the damping matrix, while the dyadic of vector $\mathbf{d}$ and its transpose, $\mathbf{d}^{T}$, contains the non-classical part resulting from the presence of the damping device. The complex eigenvalues $\lambda$ are determined as approximate solutions of characteristic equation of the system

$$
\frac{1}{\lambda}+\sum_{i=1}^{m} \frac{\tilde{d}_{i}^{2}}{\lambda^{2}+\lambda \tilde{C}_{p_{i, i}}+\omega_{i}^{2}}=0
$$

where $\tilde{C}_{p_{i, i}}=\boldsymbol{\Phi}_{i}^{T} \mathbf{C}_{p} \boldsymbol{\Phi}_{i}, \quad \tilde{d}_{i}=\boldsymbol{\Phi}_{i}^{T} \mathbf{d}, \boldsymbol{\Phi}_{i}$ is the i-th mass normalized eigenmode of undamped system and $\omega_{i}$ is the corresponding i-th real angular eigenfrequency. To approximately solve Eq. (2), a combination of perturbation and incremental methods was proposed. At first, complex eigenvalues corresponding to $\mathbf{C}_{p}$ are determined and a small increment in the damping parameter of the device is chosen. The subsequent

Ing. Stanislav Hračov, Ph.D.: Institute of Theoretical and Applied Mechanics AS CR, v.v.i.,Prosecká 76, 19000, Prague, Czech Republic, hracov@itam.cas.cz 

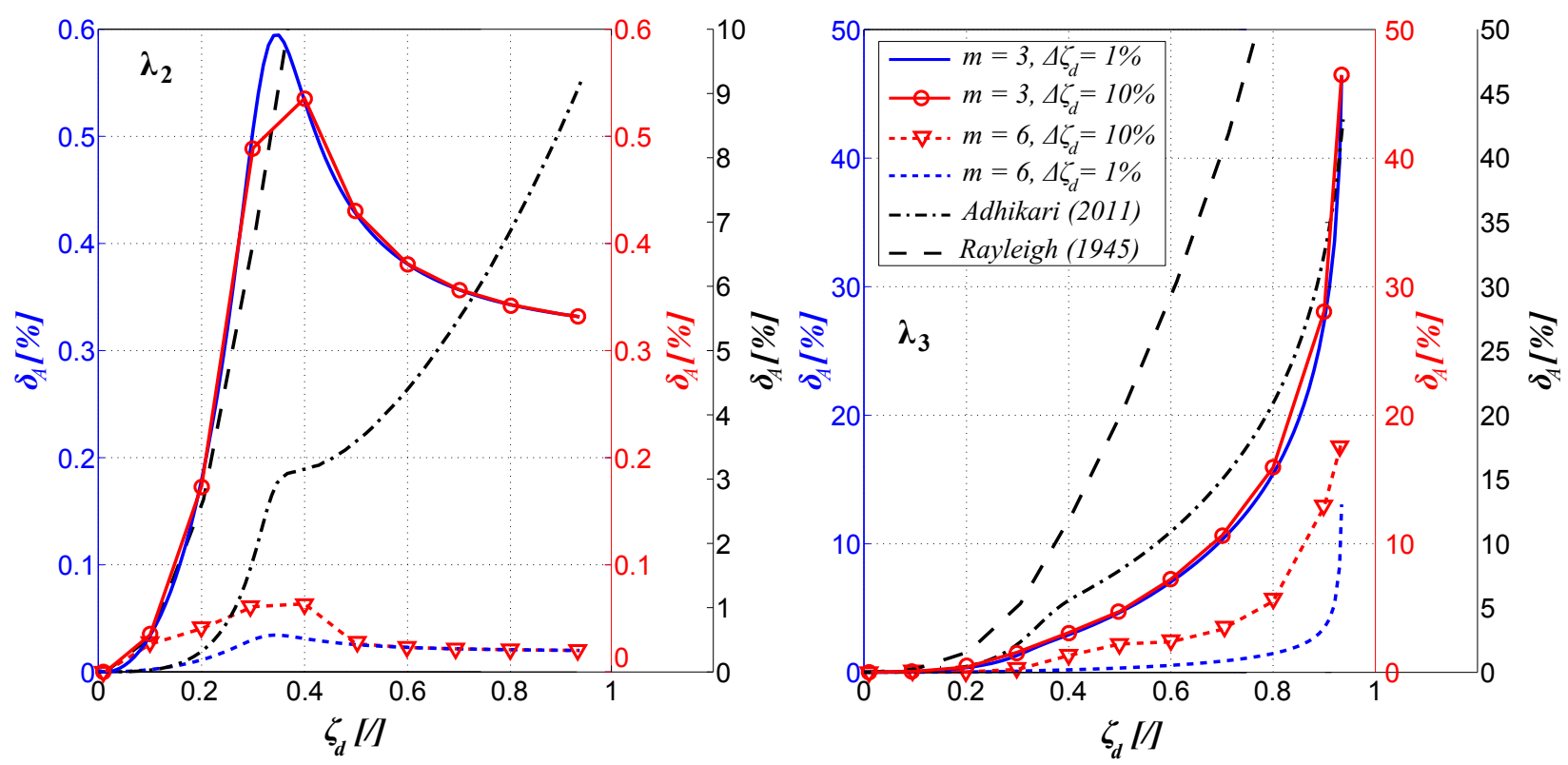

Fig. 1: The percentage relative error $\delta_{\mathrm{A}}$ of the second and third approximate eigenvalues as a function of damping ratio $\zeta_{d}$ for different numbers of eigenmodes $m$ and increments $\Delta \zeta_{d}$.

solution is divided into multiple computational steps. In each step, the damping parameter of the device is increased by the chosen small increment and the perturbation from the eigenvalue determined in the previous step is calculated. The whole computational process ends and final approximate eigensolution is obtained when the target value of the damping coefficient of the device is reached. The complex eigenmodes are subsequently calculated from the obtained eigenvalues using significantly reduced matrix system. For more details the reader is referred to the article by Hračov (2018).

\section{Analysis of accuracy and numerical efficiency of proposed method}

The accuracy of the proposed procedure was investigated on a 2D linear discrete numerical model of an existing TV tower equipped with a pendulum absorber supplemented with viscous dampers. Frequency tuning of the absorber was designed to be the most effective at vibrations in the interval of the second and third natural frequencies of the tower itself. The model was built with beam elements having 381 nodes and 1143 dofs. The absorber was modelled as one degree of freedom in the horizontal direction using a concentrated mass connected to the top of the tower with the Kelvin-Voigt damping term. The classical part, $\mathbf{C}_{p}$, of the damping matrix was assumed to be proportional to the stiffness matrix of the entire system. The structural damping ratio $\zeta$ was chosen equal to 0.005 for the lowest natural frequency. The accuracy of the proposed approximate eigensolution was investigated for a set of various damping ratios of the absorber, $\zeta_{d}$, ranging from 0 to 0.934 , while the matrix, $\mathbf{C}_{p}$, was kept constant.

The eigensolution related to individual $\zeta_{d}$ was calculated for different increments, $\Delta \zeta_{d}$, and for several numbers of natural modes, $m$, that were taken into account in the sum of the second term in Eq. (2). The modes related to the highest values of the coefficient $\alpha$, see (Claret, 1991), were selected. The accuracy of the proposed procedure was assessed by means of absolute value of percentage relative error $\delta_{A}$ corresponding to approximate eigenvalues. The accuracy generally decreases with increasing $m$ and decreasing $\Delta \zeta_{d}$. On the other hand, the time consumption of the numerical solution increases with the increasing $m$ and decreasing $\Delta \zeta_{d}$. The decreasing trend of the error $\delta_{A}$ with the increase in $m$ is apparent from Tab. 1. It is also evident in Fig. 1 from the difference between the curves with the same color. Tab. 1 and Fig. 1 also illustrate the higher accuracy of the proposed method in comparison with the approximate eigensolution proposed by Rayleigh (1945) and Adhikari (2011). Only in case of low $m$ equal to 3, the latter provided some more accurate results. The decreasing trend of the error $\delta_{A}$ with the increase in $m$ is also evident from Fig. 2, where the maximum value of $\delta_{A}$ of the third eigenvalue is plotted as a function of $m$ for several $\Delta \zeta_{d}$. It also represents the maximum error of the whole eigensolution and occured at $\zeta_{d}=0.934$. In this example, the descending trend of maximum value of $\delta_{A}$ was significant up to ten eigenmodes. Extending to more than twenty eigenmodes did not produce a substantial improvement of the accuracy, but it signifi- 
Tab. 1: The percentage relative error $\delta_{A}$ corresponding to the first four eigenvalues evaluated using two approximate methods for two values of $\zeta_{d}$ (Hračov, 2018).

\begin{tabular}{cccccccccc}
\hline \multicolumn{9}{c}{} & \multicolumn{3}{c}{ Proposed method } & \multicolumn{3}{c}{ Iterative meth. (Adhikari, 2011) } \\
$\zeta_{d}$ & & 0.1 & \multicolumn{3}{c}{0.7} & & 0.1 & 0.7 \\
$\Delta \zeta_{d}$ & & 0.01 & & \multicolumn{2}{c}{0.01} & & 0.05 & - & - \\
$m$ & 3 & & 10 & 3 & 10 & 3 & 10 & 1143 & 1143 \\
\hline$\lambda_{1}$ & $2 \cdot 10^{-4}$ & $7 \cdot 10^{-6}$ & 0.011 & $1 \cdot 10^{-4}$ & 0.011 & $1 \cdot 10^{-4}$ & 0.001 & 0.028 \\
$\lambda_{2}$ & 0.034 & $5 \cdot 10^{-4}$ & 0.357 & 0.005 & 0.357 & 0.005 & 0.028 & 5.657 \\
$\lambda_{3}$ & 0.061 & $9 \cdot 10^{-4}$ & 10.31 & 0.224 & 10.37 & 0.719 & 0.041 & 15.62 \\
$\lambda_{4}$ & 0.036 & $4 \cdot 10^{-4}$ & 0.820 & 0.011 & 0.820 & 0.011 & 0.002 & 2.033
\end{tabular}

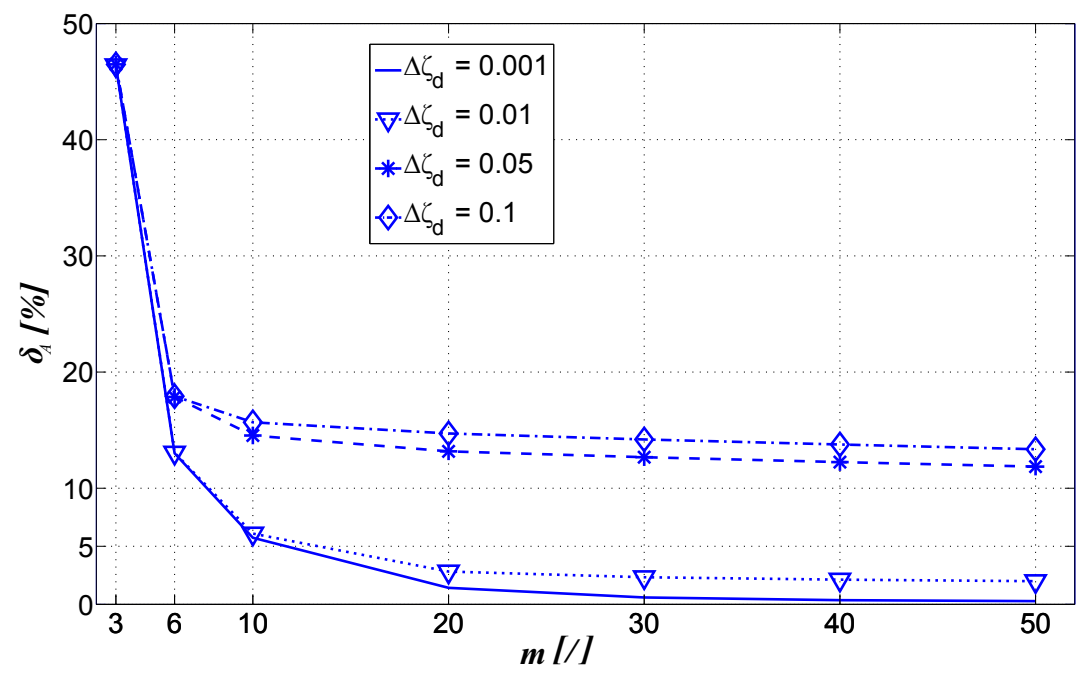

Fig. 2: The maximum value of error $\delta_{\mathrm{A}}$ of the third eigenvalue as a function of the number of natural eigenmodes $m$ for the selected increments $\Delta \zeta_{d}$.

cantly prolonged the computational time, see Tab. 2. The error $\delta_{A}$ below $10 \%$ was achieved for numerical simulations where real eigenmodes corresponding to coefficient $\alpha$ higher than 0,2 were used.

The influence of the choice of $\Delta \zeta_{d}$ on $\delta_{A}$ is apparent from the difference between the red and blue dashed curves in Fig. 1. As expected, a higher accuracy of the eigensolution was achieved for the smaller increment $\Delta \zeta_{d}$. This is also evident from the differences between the blue curves in Fig. 2 or from Tab. 1. The red and blue solid curves in Fig. 1 corresponding to low $m$ also show minimal difference between the error $\delta_{A}$ for different $\Delta \zeta_{d}$. This implies that the number of natural modes, $m$ is affecting the accuracy of the eigensolution more than increment $\Delta \zeta_{d}$. However, a too large increment $\Delta \zeta_{d}$ could also cause substantial inaccuracy, see Fig. 2. Based on this example, increment $\Delta \zeta_{d}$ equal to $1 \%$ can be recommended for such structures.

The numerical efficiency of the proposed method can also be expressed in terms of calculation time. The time required for the full eigensolution, evaluated using the state-space method, was 12.8 seconds. The proposed method provides a significantly faster eigensolution, the time of which is dependent on the required number of complex eigenmodes and eigenvalues, $n$, and on the computational parameters $m$ and $\Delta \zeta_{d}$, see Tab. 2.

\section{Acknowledgments}

The kind support of the Czech Science Foundation projects No. 17-26353J and of the RVO 68378297 institutional support are gratefully acknowledged. 
Tab. 2: Elapsed calculation time of the first $n$ complex eigenmodes and eigenvalues for two values of $\zeta_{d}$ and for the various combinations of $\Delta \zeta_{d}$ and $m$ (in seconds).

\begin{tabular}{lccccccccc}
\hline$\zeta_{d}$ & & 0.1 & & & \multicolumn{3}{c}{0.7} \\
$\Delta \zeta_{d}$ & & 0.01 & & & 0.01 & & & 0.05 & \\
$\mathrm{~m}$ & 3 & 10 & 30 & 3 & 10 & 30 & 3 & 10 & 30 \\
\hline$n=3$ & 0.031 & 0.037 & 0.163 & 0.047 & 0.095 & 1.064 & 0.032 & 0.042 & 0.243 \\
$n=6$ & 0.035 & 0.054 & 0.318 & 0.065 & 0.166 & 2.108 & 0.038 & 0.062 & 0.477 \\
$n=10$ & 0.038 & 0.065 & 0.508 & 0.085 & 0.245 & 3.480 & 0.042 & 0.079 & 0.781 \\
$n=20$ & 0.047 & 0.099 & 1.012 & 0.142 & 0.476 & 7.018 & 0.054 & 0.133 & 1.541
\end{tabular}

\section{References}

Adhikari, S. (2011) An iterative approach for nonproportionally damped systems. Mechanics Research Communications, Vol 38, pp 226-230.

Claret, A. M. and Venancio-Filho, F. (1991). A modal superposition pseudo-force method for dynamic analysis of structural systems with non-proportional damping. Earthquake Engineering and Structural Dynamics, Vol. 20, pp. 303-315.

Hračov, S. (2018) Efficient approximate eigensolution for structures equipped with a passive damping device. Journal of Structural Engineering, in print, DOI: 10.1061/(ASCE)ST.1943-541X.0002017.

Rayleigh, J. W. S. (1945) The Theory of Sound, Vol. 1, Dover Publication, New York. 